\title{
Safety and Prognosis of Transarterial Chemoembolization for Octogenarians with Hepatocellular Carcinoma
}

\author{
Hua-Ming Cheng ${ }^{1,2} \cdot$ Toshihiro Tanaka $^{1} \cdot$ Hideyuki Nishiofuku ${ }^{1} \cdot$ Yuto Chanoki $^{1}$ • \\ Katsutoshi Horiuchi $^{1} \cdot$ Tetsuya Masada $^{1} \cdot$ Shota Tatsumoto $^{1} \cdot$ Takeshi Matsumoto $^{1}$. \\ Nagaaki Marugami ${ }^{1} \cdot$ Kimihiko Kichikawa $^{1}$
}

Received: 20 February 2019/Accepted: 17 July 2019/Published online: 23 July 2019

(C) Springer Science+Business Media, LLC, part of Springer Nature and the Cardiovascular and Interventional Radiological Society of Europe (CIRSE) 2019

\begin{abstract}
Purpose The global population of the aged is escalating. The need of transarterial chemoembolization (TACE) for hepatocellular carcinoma (HCC) in patients older than 80 years is on the rise. The aim of this study was to retrospectively evaluate the safety and the prognosis of TACE in octogenarians with HCC.

Materials and Methods From January 2007 to January 2018, 86 octogenarians with HCC initially treated with TACE, who were treatment naïve or had a recurrence after surgery and/or radiofrequency ablation, were enrolled in this study. The adverse events were evaluated. The overall survival (OS) after TACE and causes of death were investigated. The prognostic factors for OS were analyzed using Cox proportional hazard models.

Results Grade 4 adverse events (according to the Common Terminology Criteria for Adverse Event version 4.0) of AST, ALT and tumor rupture were found in 8, 4 and 1 patients, respectively. There were no treatment-related deaths. The 1-, 3- and 5-year overall survival rates were $84.1 \%, 61.1 \%$ and $27.6 \%$, respectively. The overall median survival time was 38.3 months (HR 2.854, 95\% CI 32.7-43.8). 56.9\% causes of death were HCC or liver dysfunction. Multivariate analysis revealed that performance status (ECOG: 0) was an independent prognostic significant factor (95\% CI 1.103-4.573; $P=.026$ ).
\end{abstract}

Toshihiro Tanaka

toshihir@bf6.so-net.ne.jp

1 Department of Radiology, Nara Medical University, 840 Shijo-cho, Kashihara 634-8522, Japan

2 Department of Medical Imaging and Interventional Radiology, Chi-Mei Medical Center, Tainan City, Taiwan
Conclusions TACE is safe and could improve survival of octogenarians with HCC. Performance status is an important prognosis factor predicting the OS.

Keywords Hepatocellular carcinoma - Transarterial chemoembolization · Octogenarians

\section{Introduction}

Hepatocellular carcinoma (HCC) is a major malignant disease, which is the second leading cause of cancer death in the world and the fifth leading cause in Japan [1, 2]. Presently, due to an increase in life expectancy of the aged, treatments for elderly patients with HCC are a critical issue. From 2015 to 2050, the number of people aged older than 80 years is estimated to increase 3.5 -fold worldwide [3]. Year by year, the average age of HCC patients has been increasing. Currently, approximately 12,000 patients with HCC over 80 years old have been newly diagnosed and approximately 13,000 patients over 80 years old died due to HCC in Japan [4].

Previously, several papers have discussed the treatment of HCC in the elderly patients. To date, there is no consensus of the definition of "elderly," but most of the studies use the cutoff of 75 years $[5,6]$. However, the life expectancy and disability should be different between octogenarians and septuagenarians [7]. Octogenarians have a higher incidence of comorbid illness and geriatric syndromes, less muscle mass and worse performance status (PS) compared with septuagenarian. Due to these reasons, 
currently, patients over 80 years old are usually omitted in prospective clinical trials [8-11].

Transarterial chemoembolization (TACE) could be useful for the extreme aged patients due to a less invasive treatment. To the best of our knowledge, to date, there have been no research studies focused on TACE for HCC patients older than 80 years old. Therefore, indication of treatment for octogenarians with HCC is controversial and the safety and prognosis after receiving TACE remain unclear.

Based on the above background, we conducted this research study to examine the safety and prognosis of TACE in octogenarians with HCC. Factors affecting prognosis for TACE were also investigated.

\section{Materials and Methods}

\section{Patients' Eligibility}

The inclusion criteria of this study were patients with HCC over 80 years old who primarily had received TACE either as first-line therapy or as salvage therapy for recurrence after radiofrequency ablation (RFA) and/or surgical resection. Through the retrospective review from the database of interventional radiology between January 2007 and January 2018, all TACE or TAE procedures conducted for octogenarian patients with HCC were selected and patients who had received prior TACE/TAE, bland TAE without chemo-drugs or any combination therapies were excluded. The additional exclusion criteria were no available data of blood tests and contrast enhanced CTs or MRIs obtained 1 month after the TACE for evaluation of liver function and tumor response.

The diagnosis of HCC was based on typical radiological feature with reference to the European Association for the Study of the Liver (EASL Guideline) [12]. The selection of treatments of TACE was determined by the Multidisciplinary Tumor Board.

Written informed consent was obtained from all patients prior to therapy. This study protocol was in accordance with the ethical guidelines of the 1975 Declaration of Helsinki. This study was approved by the Institutional Review Board.

\section{Pretreatment Evaluation}

Liver functions including Child-Pugh score and AlbuminBilirubin (ALBI) grade, tumor stage and serum AFP level were evaluated by $\mathrm{CT}$ and blood tests obtained within 4 weeks before TACE. Sarcopenia also evaluated on the $\mathrm{CT}$, which was based on the cross-sectional muscle areas $(-29$ to $+150 \mathrm{HU})$ and normalized for patient height (area/height ${ }^{2}$ ) in patients with a different gender and body mass index $[13,14]$. PS and comorbidity before TACE were also evaluated.

\section{Treatment Modality}

A unified CT and angiography system (Angio-CT System, Infinix Activ; Canon Medical Systems) was used. TACE was performed according to a standardized protocol. A 4or 5-French catheter was introduced through the femoral artery and advanced to the celiac and superior mesenteric arteries. The tumor vascularity with hepatic arteriography was assessed. Selective catheterization of the tumor feeding artery was performed by a coaxial technique using a 1.7 to 2.0-Fr tip microcatheter. For patients receiving conventional TACE, emulsion of epirubicin solution and iodized oil (Lipiodol Ultra-Fluide; Guerbet, Villepinte, France) was infused via the feeding arteries followed by 1-mm gelatin sponge particles (Gelpart, Nippon Kayaku, Tokyo, Japan) to achieve stasis. The amounts of the lipiodol and epirubicin were determined based on tumor sizes, tumor numbers and liver function. The maximal doses of lipiodol and epirubicin were $10 \mathrm{~mL}$ and $60 \mathrm{mg}$ in a single session, respectively. For patients receiving drug-eluting beads (DEB)-TACE, $75 \mathrm{mg}$ epirubicin was loaded on one vial, $2 \mathrm{~mL}$ beads (DC Beads; BTG, London, UK) and injected until near stasis. The maximal dose of DC Beads was two vials, $4 \mathrm{~mL}$ beads.

\section{Assessment of Treatment Efficacy and Safety}

Toxicities were assessed according to the Common Terminology Criteria for Adverse Event, version 4.0 [15]. Physical examinations and blood tests were performed during the hospital stay for 7 days after TACE. CT was obtained 7 days after TACE to check for adverse event. The tumor response was evaluated according to the modified Response Evaluation Criteria in Solid Tumors (mRECIST) on contrast enhanced CT obtained 1 month after TACE [16, 17]. Serum alpha-fetoprotein (AFP) assays also evaluated 1 month after TACE. If there was no viable tumor after TACE, patients were followed-up every 3 month.

Additional TACE was performed when recurrence developed. Survival duration was defined as the time from the date of the first TACE until the date of death. The causes of death were investigated.

\section{Statistical Analysis}

Statistical analyses were conducted using SPSS, version 24.0 statistical software (SPSS, Inc.; Chicago, IL, USA). The overall survival was evaluated using the Kaplan-Meier 
method, and 95\% confidence intervals (CIs) were provided for proportions. The curves were compared by log-rank test. The adverse events in conventional TACE and DEBTAE were compared by Chi-square test. Univariate and multivariate Cox regression analyses were performed to assess prognostic factors of overall survival. Multivariate analysis was undertaken for variables demonstrating $P<0.10$ after univariate analysis, and $P<0.05$ was considered statistically significant.

\section{Results}

\section{Patients' Characteristics}

A total of 207 patients aged over 80 years received TACE or TAE. One hundred and five patients were excluded; 92 had prior TACE before this study period, and 13 had received bland embolization or combination therapy with intra-arterial infusion. Sixteen patients were excluded due to no record of blood tests in this institute, and/or no contrast enhanced CTs or MRIs 1 month after TACE. Finally, 86 patients were included in this study (Fig. 1). Thirty-nine $(62.9 \%)$ men and $16(66.6 \%)$ women diagnosed with sarcopenia. Seventy-eight patients received conventional TACE and the remaining eight received DEB-TACE due to the discretion of the operator. The baseline clinical and demographic characteristics of patients are shown in Table 1.

\section{Response and Adverse Events}

Complete response (CR) was achieved in 60 patients (69.8\%), partial response (PR) in $22(25.6 \%)$, stable disease in two and progression disease in two. The response rate $(\mathrm{CR}+\mathrm{PR})$ was $95.3 \%$. All adverse events are illustrated in
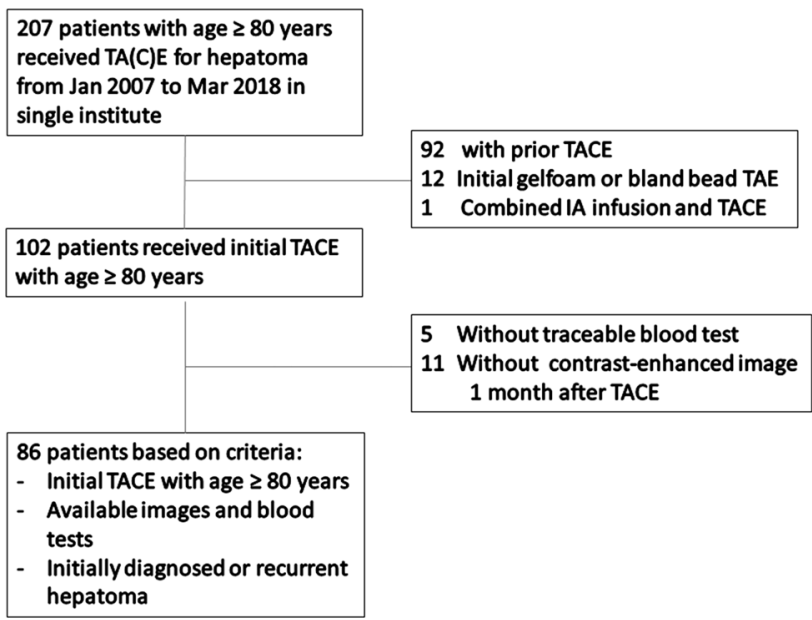

Fig. 1 Flow diagram shows patient selection criteria
Table 1 Patients' baseline characteristics $(n=86)$

\begin{tabular}{|c|c|}
\hline Variables & Values \\
\hline Mean and median age ( $\left.{ }^{\mathrm{a}} \mathrm{SD} ;{ }^{\mathrm{b}} \mathrm{IQR}\right)$ & $82\left({ }^{\mathrm{a}} 2.098829 ;{ }^{\mathrm{b}} 80-83\right)$ \\
\hline \multicolumn{2}{|l|}{ Gender } \\
\hline Male & 62 \\
\hline Female & 24 \\
\hline \multicolumn{2}{|l|}{ ECOG performance status } \\
\hline 0 & 60 \\
\hline $1-2$ & 24 \\
\hline $3-4$ & 2 \\
\hline \multicolumn{2}{|l|}{ BCLC classification } \\
\hline A & 41 \\
\hline B & 15 \\
\hline $\mathrm{C}$ & 28 \\
\hline $\mathrm{D}$ & 2 \\
\hline \multicolumn{2}{|l|}{ Child-Pugh } \\
\hline A & 83 \\
\hline $\mathrm{B}$ & 3 \\
\hline \multicolumn{2}{|l|}{ Etiology of $\mathrm{HCC}$} \\
\hline Hepatitis B virus (HBV) & 4 \\
\hline Hepatitis $\mathrm{C}$ virus (HCV) & 46 \\
\hline $\mathrm{HBV}+\mathrm{HCV}$ & 9 \\
\hline Alcoholic & 7 \\
\hline \multicolumn{2}{|l|}{ Comorbidity } \\
\hline Hypertension & 58 \\
\hline $\mathrm{DM}$ & 31 \\
\hline Other cancer & 20 \\
\hline Old myocardial infarct & 14 \\
\hline Cerebral infarction & 8 \\
\hline \multicolumn{2}{|l|}{ Treatment prior to TACE } \\
\hline None & 53 \\
\hline RFA only & 10 \\
\hline Surgery only & 19 \\
\hline Both RFA and surgery & 4 \\
\hline \multicolumn{2}{|l|}{ Tumor number in each patient } \\
\hline$<3$ & 71 \\
\hline$\geq 4$ & 15 \\
\hline \multicolumn{2}{|l|}{ Portal venous tumor thrombus (PVTT) } \\
\hline No & 81 \\
\hline Yes & 5 \\
\hline \multicolumn{2}{|l|}{ Extrahepatic metastasis } \\
\hline No & 82 \\
\hline Yes & 4 (3 lung; 1 spine) \\
\hline
\end{tabular}

Variables are expressed as median (interquartile range)

${ }^{\mathrm{a}} S D$ standard deviation; ${ }^{\mathrm{b}} I Q R$ interquartile range

Table 2. There were no treatment-related deaths. There were no 30-day and in-hospital mortality cases. A total of 77 grade 3 and 4 adverse events occurred; however, many 
Table 2 Adverse events observed after embolization

\begin{tabular}{lrrrrrl}
\hline Adverse event $^{\mathrm{a}}$ & \multicolumn{3}{c}{ CTCAE grade } & \multicolumn{2}{l}{$\begin{array}{l}\text { Frequency; } \\
n(\%) \geq \text { grade } 3\end{array}$} \\
\cline { 2 - 5 } & \multicolumn{1}{c}{1} & \multicolumn{1}{c}{3} & 3 & 4 & 5 & \\
\hline Ascites & 7 & 1 & 0 & 0 & 0 & $0(0 \%)$ \\
Pleural effusion & 8 & 7 & 0 & 0 & 0 & $0(0 \%)$ \\
Bile duct injury & 0 & 3 & 1 & 0 & 0 & $1(1.2 \%)$ \\
Tumor rupture & 0 & 0 & 0 & 1 & 0 & $1(1.2 \%)$ \\
ALP & 4 & 6 & 1 & 0 & 0 & $1(1.2 \%)$ \\
ALT & 28 & 18 & 26 & 4 & 0 & $30(34.9 \%)$ \\
AST & 25 & 12 & 34 & 8 & 0 & $42(48.8 \%)$ \\
Albumin & 47 & 35 & 1 & 0 & 0 & $1(1.2 \%)$ \\
Bilirubin & 29 & 15 & 0 & 0 & 0 & $0(0 \%)$ \\
Abdominal pain & 25 & 12 & 1 & 0 & 0 & $1(1.2 \%)$ \\
Pyrexia & 43 & 9 & 0 & 0 & 0 & $0(0 \%)$ \\
Nausea & 33 & 19 & 0 & 0 & 0 & $0(0 \%)$ \\
Vomiting & 11 & 3 & 0 & 0 & 0 & $0(0 \%)$ \\
Fatigue & 33 & 1 & 0 & 0 & 0 & $0(0 \%)$
\end{tabular}

$\overline{A L P}$ alkaline phosphatase, $A L T$ alanine aminotransferase, $A S T$ aspartate aminotransferase

${ }^{\mathrm{a} C}$ Common Terminology Criteria for Adverse Events (CTCAE)

of them were serum aspartate aminotransferase (AST) and alanine aminotransferase (ALT) elevations. One patient $(1.2 \%)$ had severe abdominal pain after TACE. One patient $(1.2 \%)$ developed tumor bleeding after DEB-TACE. The tumor was located subcapsular of the liver and vascular lake appeared during DEB-TACE; however, it was not embolized due to lack of knowledge relating to the management of vascular lake. This was our first case involving vascular lake. Consequently, intraabdominal hemorrhage occurred 2 days after TACE, which required emergent TAE by gelatin particles. The other patient needed percutaneous biliary drainage due to bile duct injury after conventional TACE. Over grade 3, ALT elevation occurred in one patient $(11 \%)$ and AST elevation in two (22\%) after DEB-TACE, while ALT elevation occurred in 29 (37.7\%) and AST elevation in 40 (51.9\%) after conventional TACE. All grade 4 AST and/or ALT elevation occurred in conventional TACE (both AST and ALT 3, ALT alone 1 and AST alone 5). Sever AST elevation tended to occur more frequently after conventional TACE compared with DEBTACE although there were no statistically significant differences $(P=0.089)$. There were no deteriorations of the comorbidities by TACE.

\section{Follow-Up, Overall Survival and Cause of Death}

Forty-one patients received one treatment of TACE and the remaining 45 received additional TACE after recurrence; two times TACE in 19 patients, 3-5 times in 21 and over six times in five. The overall survival is illustrated in Fig. 2. The overall median survival time was 38.3 months (95\% CI 32.7-43.8). The 1-, 3- and 5-year survival rates were $84.1 \%, 61.1 \%$ and $27.6 \%$, respectively. Fifty-eight patients died during this follow-up period. Thirty-three $(56.9 \%)$ died due to liver-related disease, HCC or liver cirrhosis, and the remaining 25 died due to other causes (Table 3).

\section{Univariate and Multivariate Analysis of Predictive Factors for Patient Survival}

Table 4 shows the factors associated with overall survival. In univariate analysis, performance status $0(95 \%$ CI $0.934-3.542 ; P=0.079)$, Child-Pugh class A $(95 \%$ CI 1.106-20.891; $P=0.036)$, serum AFP level $<200(95 \%$ CI $1.091-4.970 ; P=0.029)$, ALBI grade $1(95 \%$ CI $0.957-3.726 ; P=0.067)$ and complete response $(95 \% \mathrm{CI}$ $1.033-3.512 ; P=0.039)$ were the positive prognostic factors. There was no statistically significant association between the overall survival and coexisting comorbidity including hypertension, DM, COPD, cerebral infarction and second cancer. Also, sarcopenia did not influence the survival. Multivariate analysis showed that the performance status was the only predictive factor showing statistically significant (95\% CI 1.089-4.480; $P=0.026$ ).

Figure 2 shows the Kaplan-Meier curves of overall survival according to performance status divided into zero and nonzero. In patients with PS-0, the median survival was 43.2 months, whereas that of 29.3 months in patients with $\mathrm{PS} \geq 1$. There was a significant difference between them $(P=0.005)$.

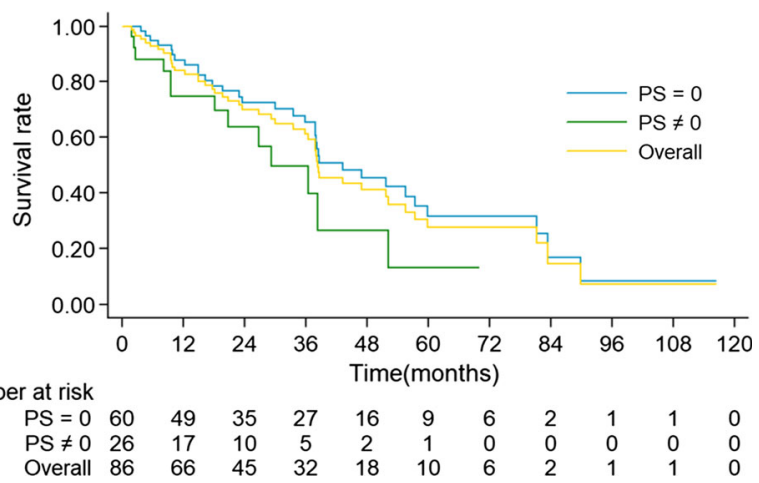

Fig. 2 Cumulative survival curves of overall (yellow color), PS = 0 (blue color) and PS $\geq 1$ (green color) 
Table 3 Causes of death of 58 died patients

\begin{tabular}{lc}
\hline Causes & Number \\
\hline HCC & 23 \\
Liver failure & 10 \\
Pneumonia & 3 \\
Upper gastrointestinal hemorrhage & 2 \\
Intracranial hemorrhage & 2 \\
Subarachnoid hemorrhage & 1 \\
Cerebral infarction & 1 \\
Lung cancer & 1 \\
Interstitial pneumonia & 1 \\
Acute respiratory distress syndrome & 1 \\
Acute cardiac death & 1 \\
Low blood sugar, adrenal failure & 1 \\
Old age & 11 \\
\hline
\end{tabular}

\section{Discussion}

Recently, research studies regarding treatments' safety and outcome of cancer patients aged over 80 years are trend due to the increase number of extreme aged patients worldwide. The studies of prostate cancer, breast cancer, colorectal cancer and gastric cancer have been published [8-11].

This study indicated the safety of TACE for HCC patients older than 80 years. Previously, the results of PRECISION V study showed that more than 20 serious adverse events (SAE) occurred after TACE in 200 enrolled patients with the mean age of 67 years old [18]. We experienced only 2 SAE in 86 patients, although our retrospective study might not evaluate postembolization syndrome, i.e., pain or nausea, correctly. There were no treatment-related deaths and no deteriorations of the comorbidities by TACE.

Previously, Ikeda et al. reported the result of TACE for HCC in an Asian cooperative prospective study [19]. The median age of the enrolled patients was 70 (range 45-84), and the median survival rate was 36 months. In this study, the overall survival period was 38.3 months, which seemed to be comparable to that of younger patients in Ikeda's report. The multivariate analysis showed patients with poor PS had significantly shorter overall survival. Octogenarians more frequently had worse PS than septuagenarians. In our study, $30 \%$ of the enrolled patients had performance status of 1-3. Previously, Kaibori et al. [6] reported that, in a Japanese Nationwide Cohort, approximately $15 \%$ of over 75-year-old HCC patients had PS 1-3. The balance between treatment effect and life expectancy has to be considered before TACE in extreme aged patients with poor PS.

To the best of our knowledge, there has been only one published literature in which the outcome of TACE for elderly HCC patients was examined by Nishikawa et al. [5]. They evaluated patients over 75 years old and the OS of 34 months and 1- and 3-year survival rates of $84 \%$ and $48 \%$, respectively. They evaluated only pretreatment liver function and tumor stage, not PS for prognostic factors, and
Table 4 Prognostic factors predicting overall survival in octogenarians with hepatocellular carcinoma undergoing TACE

\begin{tabular}{llllll}
\hline Variables & \multicolumn{2}{l}{ Univariate analysis } & & \multicolumn{2}{l}{ Multivariate analysis } \\
\cline { 2 - 3 } & $P$ value & $95 \%$ CI & & $P$ value & $95 \%$ CI \\
\hline Age (82 years) & 0.566 & $0.636-2.288$ & & \\
Gender (M/F) & 0.412 & $0.678-2.575$ & & \\
Performance status (0) & 0.079 & $0.934-3.542$ & & 0.026 & $1.103-4.573$ \\
Sarcopenia & 0.266 & $0.764-2.648$ & & \\
Child-Pugh class (A) & 0.036 & $1.106-20.891$ & & 0.123 & $0.726-14.755$ \\
AFP (> 200 ng/ml) & 0.029 & $1.091-4.970$ & & 0.117 & $0.840-4.738$ \\
ALBI grade (1) & 0.067 & $0.957-3.726$ & & 0.090 & $0.908-3.804$ \\
Complete response as the initial response & 0.039 & $1.033-3.512$ & & 0.079 & $0.934-3.479$ \\
Hypertension & 0.680 & $0.598-2.200$ & & \\
DM & 0.294 & $0.369-1.351$ & & \\
Second cancer & 0.469 & $0.670-2.390$ & & \\
COPD & 0.272 & $0.661-4.365$ & & \\
Cerebral infarction & 0.523 & $0.532-3.461$ & & \\
BMI (> 25) & 0.854 & $0.539-2.110$ & & \\
Prior liver surgery & 0.371 & $0.342-1.492$ & & \\
Prior RFA & 0.840 & $0.461-2.596$ & & \\
\hline AFP & &
\end{tabular}

$A F P$ alpha-fetoprotein; $A L B I$ albumin-bilirubin, $B M I$ body mass index; $R F A$ radiofrequency ablation 
reported that Child-Pugh class and tumor number and size were significant factors.

The presence of sarcopenia was considered to be a prognosis predictive factor in cancer patients in previous studies $[13,20,21]$. In our study, 55 patients $(62.9 \%)$ were sarcopenic. The prevalence of sarcopenia of these patients had similar result as those in the previous study by Yamada et al. [22]. The overall survival of patients with sarcopenia in our study did not show any statistically significant differences compared to ones without sarcopenia $(P$ value $=0.266)$. The reason sarcopenia is not a prognosis indicator is considered to be due to small sample size or influences by various other factors, i.e., liver function.

The complete response in the initial TACE was not a positive prognostic factor in the multivariate analysis although it was shown in the univariate analysis. This could also be due to small sample size [17].

In this study, the cause of death of $56.9 \%$ of patients was related to HCC instead of other systemic or geriatric diseases. Although Japan is one of the world's highest ranking countries for longevity, the life expectancy of Japanese men is limited to 80.5 years [23]. Our data showed the probability to prolong survival of HCC patients over 80 years old by TACE.

In this study, conventional TACE and DEB-TACE were included. Previous reports showed DEB-TACE showed less sever AST and ALT elevations compared with conventional TACE [24]. Our study also showed similar results, $11 \%$ and $12 \%$ versus $37 \%$ and $52 \%$, respectively, although there was no significant difference due to small patient number in DEB-TACE. Postembolization syndrome also seemed to be worse in conventional TACE.

There are several limitations to this study. First, the number of patients included in this study is limited. Second, this was not a comparative study. Third, there could be selection bias regarding the indication of TACE since this was a retrospective study. To clarify the risk and no inferiority outcome of TACE for extreme elderly aged patients, a prospective comparative study is needed.

In conclusion, TACE is safe and could prolong survival for octogenarians with HCC. PS is an individual important prognosis factor for predicting the overall survival. It is worth considering the indication of TACE for extreme aged HCC patients.

Acknowledgements We thank Ms. Marian Pahud for advice in submitting this article.

\section{Compliance with Ethical Standards}

Conflict of interest All authors have no conflicts of interest and financial disclosures to declare.
Ethical Approval Ethical Committee in our institution approved this study.

Informed Consent Informed Consent Statement was obtained by each enrolled patient.

\section{References}

1. Dimitroulis D, Damaskos C, Valsami S, et al. From diagnosis to treatment of hepatocellular carcinoma: an epidemic problem for both developed and developing world. World J Gastroenterol. 2017;23:5282.

2. Matsuda T, Marugame T, Kamo KI, Katanoda K, Ajiki W, Sobue T, The Japan Cancer Surveillance Research Group. Cancer incidence and incidence rates in Japan in 2005: based on data from 12 population-based cancer registries in the Monitoring of Cancer Incidence in Japan (MCIJ) Project. Jpn J Clinl Oncol. 2011;41:139-47.

3. He W, Goodkind D, Kowal P. An aging world: 2015. Washington, DC: U.S. Government Publishing Office, 2016. https://www. census.gov/content/dam/Census/library/publications/2016/demo/ p95-16-1.pdf. Accessed 13 Dec 2016.

4. National Cancer Center, Japan. https://ganjoho.jp/reg_stat/ statistics/stat/summary.html.

5. Nishikawa H, Kita R, Kimura T, et al. Transcatheter arterial chemoembolization for intermediate-stage hepatocellular carcinoma: clinical outcome and safety in elderly patients. J Cancer. 2014:5:590-7.

6. Kaibori M, Yoshii K, Hasegawa K, et al. Treatment optimization for hepatocellular carcinoma in elderly patients in a Japanese nationwide cohort. Ann Surg. 2019;270(1):121-30.

7. WHO Global Health Observatory (GHO) data. http://www.who. int/gho/mortality_burden_disease/life_tables/situation_trends/en/.

8. Masaoka H, Ito H, Yokomizo A, Eto M, Matsuo K. Potential overtreatment among men aged 80 years and older with localized prostate cancer in Japan. Cancer Sci. 2017;108:1673-80.

9. Glaser R, Marinopoulos S, Dimitrakakis C. Breast cancer treatment in women over the age of 80: a tailored approach. Maturitas. 2018;110:29-32.

10. Kang T, Kim HO, Kim H, Chun HK, Han WK, Jung KU. Age over 80 is a possible risk factor for postoperative morbidity after a laparoscopic resection of colorectal cancer. Ann Coloproctol. 2015;31:228.

11. Park HJ, Ahn JY, Jung HY, et al. Clinical characteristics and outcomes of gastric cancer patients aged over 80 years: a retrospective case-control study. PLoS ONE. 2016;11:e0167615.

12. Galle PR, Forner A, Llovet J, et al. EASL Clinical practice guidelines: management of hepatocellular carcinoma. J Hepatol. 2018;69:182-236.

13. Begini P, Gigante E, Antonelli G, et al. Sarcopenia predicts reduced survival in patients with hepatocellular carcinoma at first diagnosis. Ann Hepatol. 2017;16:107-14.

14. Martin L, Birdsell L, Macdonald N, et al. Cancer cachexia in the age of obesity: skeletal muscle depletion is a powerful prognostic factor, independent of body mass index. J Clin Oncol. 2013;31:1539-47.

15. Common Terminology Criteria for Adverse Events v.4.0 (CTCAE). NIH publication \#09-7473. National Cancer institute. http://ctep.cancer.gov/protocolDevelopment/electronic_applications/ ctc.htm. 2009. Accessed 1 Mar 2013.

16. Lencioni R, Llovet J. Modified RECIST (mRECIST) assessment for hepatocellular carcinoma. Semin Liver Dis. 2010;30:052-60.

17. Kim BK, Kim SU, Kim KA, et al. Complete response at first chemoembolization is still the most robust predictor for favorable 
outcome in hepatocellular carcinoma. J Hepatol. 2015;62:1304-10.

18. Lammer J, Malagari K, Vogl T, et al. Prospective randomized study of doxorubicin-eluting-bead embolization in the treatment of hepatocellular carcinoma: results of the PRECISION V study. Cardio Vasc Interv Radiol. 2010;33:41-52.

19. Ikeda M, Arai Y, Park SJ, et al. Prospective study of transcatheter arterial chemoembolization for unresectable hepatocellular carcinoma: an Asian cooperative study between Japan and Korea. J Vasc Intervent Radiol. 2013;24:490-500.

20. Chang KV, Chen JD, Wu WT, Huang KC, Hsu CT, Han DS. Association between loss of skeletal muscle mass and mortality and tumor recurrence in hepatocellular carcinoma: a systematic review and meta-analysis. Liver Cancer. 2018;7:90-103.

21. Ha Y, Kim D, Han S, et al. Sarcopenia predicts prognosis in patients with newly diagnosed hepatocellular carcinoma, independent of tumor stage and liver function. Cancer Res Treat. 2018;50:843-51.

22. Yamada M, Nishiguchi S, Fukutani N, et al. Prevalence of sarcopenia in community-dwelling Japanese older adults. J Am Med Dir Assoc. 2013;14:911-5.

23. Japan Ministry of Internal Affairs and Communications, Statistics Bureau. http://www.stat.go.jp/data/topics/topi721.htm. Accessed 15 Sept 2013.

24. Vogl TJ, Lammer J, Lencioni R, et al. Liver, Gastrointestinal, and cardiac toxicity in intermediate hepatocellular carcinoma treated with PRECISION TACE with drug-eluting beads: results from the PRECISION V randomized trial. AJR. 2011;197:W562-70.

Publisher's Note Springer Nature remains neutral with regard to jurisdictional claims in published maps and institutional affiliations. 